\title{
FIXATION FREE INTER-POSITIONAL MANDIBULAR GRAFTING A NOVEL TECHNIQUE FOR ALVEOLAR RIDGE HEIGHT AUGMENTATION VERSUS THE CONVENTIONAL FIXATION TECHNIQUE. (RANDOMIZED CONTROL CLINICAL TRIAL)
}

\author{
Hesham S. Abdelmoneim*, Mohamed Ashraf* and Radwa T. Elsharkawy**
}

\begin{abstract}
The aim of this study is to determine whether the technique of interpositional grafting of the posterior mandible without the use of fixation device (using bone blocks alone) is superior to the conventional interpositional grafting with fixation (plates and screws). This study was conducted on 15 patients in which 16 mandibular segments were augmented, segments were randomly divided into 2 groups, control receiving interpositional segmental grafting of the posterior mandible with fixation and intervention group receiving same procedure but without grafting and fixed via chin bone blocks. Results revealed better overall clinical and radiographic results toward the intervention group in terms of graft resorption and application but was statistically non-significant, while the histological evaluation yielded to the postulation that no microscopic difference between both techniques in terms of the resultant bone. Thus concluding that interpositional grafting technique without the use of a fixation device was a reliable technique for vertical ridge augmentation of the atrophied posterior mandibular ridges with promising rates of success, reduced complications and reduced bone resorption values compared to conventional interpositional grafting with fixation protocol.
\end{abstract}

KEYWORDS Interpositional grafting, inlay graft, fixation free interpositional graft, vertical ridge augmentation.

\section{INTRODUCTION}

Rehabilitation of partially or totally edentulous mandibles with implant supported prosthesis has become a common alveolar ridge reconstruction surgery, with near reliable long term outcomes. Edentulous ridges local conditions may be unfavorable for dental implants installation, where sufficient alveolar or basal bone foundation along both the horizontal and vertical dimensions is considered to be the foundation for a successful oral rehabilitation process with dental implants. ${ }^{(1,2)}$

Obstructing or hindering factors include the

* Lecturer of Oral and Maxillofacial Surgery, Faculty of Dentistry, Cairo University.

** Lecturer of Oral Biology, Faculty of Dentistry, Future University in Egypt. 
bony resorptive changes of the alveolar ridge after dental extractions, in addition to the hard tissue deficits that could result from chronic periodontal diseases or congenitally missing dentition. Neoplasms, osteomyelitis, cysts even trauma could compromise the maxillary and mandibular ridges status. All stated factors usually imply the dental implantologist to utilize regenerative techniques to gain a sufficient hard tissue base suitable for dental implants installation. ${ }^{(1,2)}$

Several bone grafting materials and techniques have been implemented to reconstruct the partially and totally edentulous alveolar ridges with relatively high rates of success. Those techniques include block onlay grafting, inlay grafting, guided bone regeneration using membranes with or without meshes, inferior alveolar nerve lateralization, inferior alveolar nerve transposition and distraction osteogenesis. Optimal technique selection depends on various factors, including the magnitude of the defect, the grafted bone substitute material available, the medical status of the patient and also the skill and experience of the surgeon. ${ }^{(3-5)}$

When a particulate graft is selected for vertical or transverse (horizontal) augmentation, a rigid membrane or mesh must be used to stabilize the graft. The process of vertical augmentation with particulate grafts stabilized by a rigid membrane was reported to be successful. These techniques have been usually associated with high rates of graft exposure and resorption rates that can negatively affect the grafting procedure. Exposure rates were reported to be as high as $50 \%$, particularly when large vertical augmentations were performed. ${ }^{\left({ }^{(}\right)}$

The rationale of any grafting procedure is to maximize the blood supply to the underlying bone graft and to prevent hypoxia with resulting ischemic changes at the distal portions of the flap which could eventually lead to surgical wound dehiscence. ${ }^{(7,8)}$ The most frequently reported and feared from complications with bone grafting in literature are surgical wound dehiscence, exposure of the grafts and subsequent grafted bone resorption. $(9,10)$

It has been stated that Interpositional grafting has the advantage of ensuring good vascularity to the graft, which should in turn result in lower resorption. There by the mobilized segment usually remains dimensionally stable because of the sustained periosteal blood supply, in addition to the graft endosteal incorporation from the adjacent bone marrow. ${ }^{(11)}$

Visor osteotomy, a segmental osteotomy that's grafted interpositionally, first described in 1975 by Harle $^{(12)}$. In 1976, Schettler and Holtermann ${ }^{(13)}$ stated sandwich technique in the anterior mandible, which consisted of a parasagital split of the mandible, from mid body on one side to the same position on the contralateral side, with the inner segment of bone is pedicled to the lingual mucoperiostium.

In a research by Scarano A. et al ${ }^{(1)}$ aiming to augment atrophic posterior mandible using an inlay technique with a xenograft without the use of miniscrews or miniplates, stating that the alveolar bone height was increased immediately after surgery to an average gain of $14.15 \mathrm{mms}$. The median loss of vertical bone height after grafting and during the 4 months after surgery was $2.45 \mathrm{mms} \pm 0.3 \mathrm{mms}$. Thus concluding that interpositional bone grafting in the posterior mandible was a reliable augmentation procedure with promising results.

The use of mini screws and plates for the stabilization of the elevated segment could result in the fracture of the osteomized segments. This fracture could be responsible for bone resorption and thus failure of the technique, which in turn pools into the advantage of the use of interpositional grafting without fixation to stabilize and immobilize the elevated bony segments. ${ }^{(14)}$

There by the aim of that study was to evaluate whether chin (autogenous) bone blocks could be 
used as an interpositional grafting method of the deficient alveolar mandibular ridges without the use of a fixation device and could be an alternative to the use of mini plates and screws.

\section{MATERIALS AND METHODS}

This study was a double blinded, parallel categorized randomized controlled clinical trial. Patients of this study were allocated into 2 groups A (control) and B (intervention). This study was conducted on 15 patients in which 16 mandibular segments were augmented, segments were divided into 2 groups each consisting of 8 segments. Inclusion criteria set that patients should be free from any systemic condition that might affect the proposed outcomes, or even contraindicate the surgical procedures, edentulous posterior mandibular region (premolar/molar) with no history of previous attempts for grafting of the vertically deficient alveolar ridge, vertical deficiency of the alveolar bone height less than $8 \mathrm{mms}$ and more than $4 \mathrm{mms}$ but with sufficient width (horizontal dimension) of the alveolar ridge. Through a buccal para-crestal incision the deficient alveolar segment to be augmented was exposed, then osteotomy cuts were performed initially using surgical ridge splitting discs then the osteotomy was completed

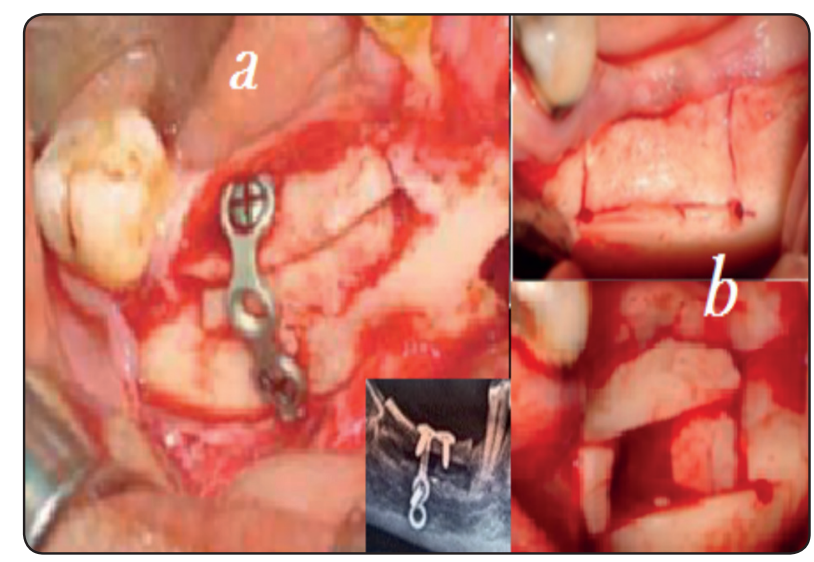

Fig. (1) a- showing group A segments with the osteotomy, graft placed and fixation using mimi-plates and screws. bShowing the osteotomy and alveolar segment elevation with Mini Blocks (group B) to lingual via ridge splitting chisels, leaving the lingual mucoperiosteium non reflected and attached to the alveolar segment. After complete freeing of the osteotomized segment (pedicled on the lingual mucoperiostieum) it was elevated and fixed to the desired height via mini plates and screws for segments of group A (fig.1a), while group B segments were stabilized by wedging the prepared chin blocks along the mesial and distal ends of the elevated segment without the use of any fixation device (fig.1b). The harvested chin blocks were cut to the required dimensions (fig.2) to attain the required augmentation height. The gap under the elevated segments of both groups were obliterated via autogenous bone particulates harvested from the chin area followed by collagen membrane placement over the osteotomy site then the closure of the surgical wound. After 4 months the grafted area was revisited in group A only to remove the fixation device (plates and screws) The augmented segments were evaluated clinically for dehiscence, mobility (digital testing) and overall success of the augmentation procedure at 1 week, 1 month and 4 months post operative. Radiographic evaluation by a series of CBCTs made pre-operatively, immediately post-operative and 4 months postoperative. Radiographic stent was fabricated for

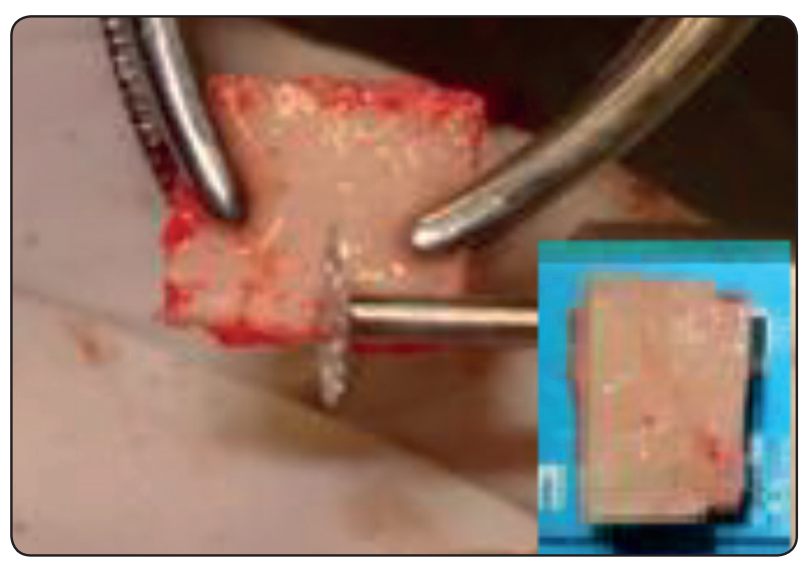

Fig. (2) Showing harvested chin blocks prepared to required dimentions. 
each case and measurements were taken from the CBCT under the radio-opaque markers of the radiographic stent from the crest of the ridge to the superior margin of the inferior alveolar canal. The immediately post operative measurements were subtracted from the pre operative measurements to determine the amount of augmentation gained and the 4 months measurements were subtracted from the immediately post operative measurements to determine the amount of bone resorption occurred after 4 months healing period (fig.3). The obtained numbers were then converted to percentile to allow for the evaluation and comparison of the results of the two procedures, where the percentile of the amount of augmentation was to the actual preoperative ridge height, while the percentile of the resorption was to the amount of augmentation attained.

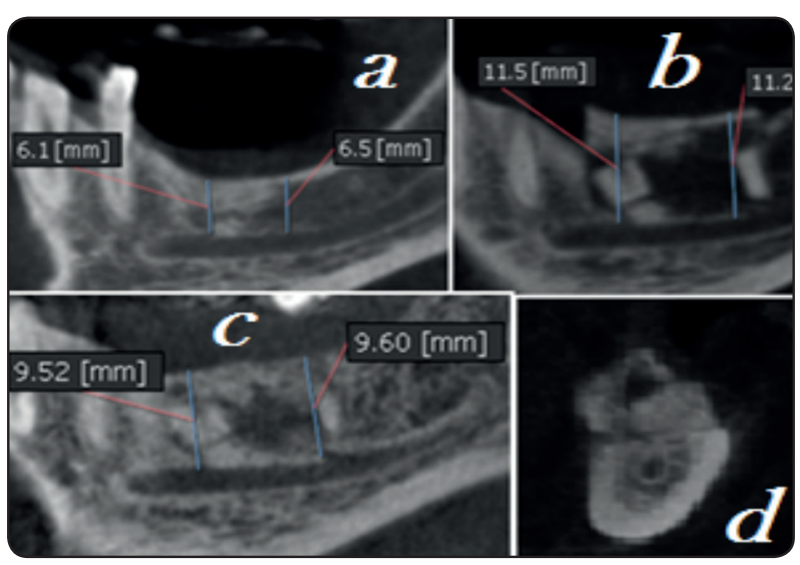

Fig. (3) Showing; a- preoperative measurements taken under radio-opaque markers of the ridge height. bimmediately post operative measurements. c- 4 months post operative measurements. $d$ - cross section of the augmented area of a group B case showing the mini block graft in place.

Statistical analysis was performed to the obtained results using Microsoft excel statistical analyzer, AVERAGE, STDEV and $\mathrm{P}$ value calculated by $\mathrm{T}$ test to compare variables between the two groups. The results were considered statistically significant if the $\mathrm{p}$ value was $\leq 0.05$.
Histological evaluation was done on core biopsy taken from the site of dental implant osteotomy via a $3 \mathrm{~mm}$ external diameter trephine bur at 4 months post-operative. The biopsy specimens were fixed immediately in $10 \%$ neutral buffered formalin, then after complete decalcification, specimens were processed, infiltrated in paraffin wax and embedded in the center of wax blocks. The specimens were cut into $5 \mu \mathrm{m}$ thick sections which were then stained by Hematoxylin and Eosin (H\&E) stain. After fixation, specimens were washed under running water, dehydrated by transferring through serial ascending concentrations of alcohol. The stained sections were then examined under a light microscope (Trinocular microscope Olympus, BX46).

\section{RESULTS}

\section{Clinical evaluation results (table 1)}

Clinical evaluation of both groups resulted in a total of 2 cases of group A (fixation technique) which suffered from surgical wound dehiscence after 2 and 3 weeks respectively post-operative with subsequent exposure of the fixation devices, both cases showed signs of infection which were resistant to antibiotics and local irrigation and antiseption trials, which thereby required removal of the fixation device, so were considered to be failed cases and rolled out of the statistics. Also one of the segments experienced a mal fracture of the segment which required the use of a mini screw to stabilize it and correct it. On the other hand group B showed surgical wound dehiscence in one the cases which was managed by antibiotics and oral hygiene measures and the wound was completely closed after 1 month post-operative (fig.4), thereby it was not eliminated from the research.

Mobility testing by digital pressure of the segments to check for signs of loss of stability postulated that all the cases of both groups were stable throughout the follow up intervals. 
Overall success revealed revealed a failure of 2 cases of the group A segments and 1 case of infection of group B segments.

\begin{tabular}{|c|c|c|c|c|}
\hline Table 1 & Dehiscence & Infection & $\begin{array}{c}\text { Survival } \\
\text { (success) }\end{array}$ & Mobility \\
\hline $\begin{array}{c}\text { A (8 } \\
\text { segments) }\end{array}$ & 2 & 2 & 6 & 0 \\
\hline $\begin{array}{c}\text { B (8 } \\
\text { segments })\end{array}$ & 1 & 1 & 8 & 0 \\
\hline
\end{tabular}

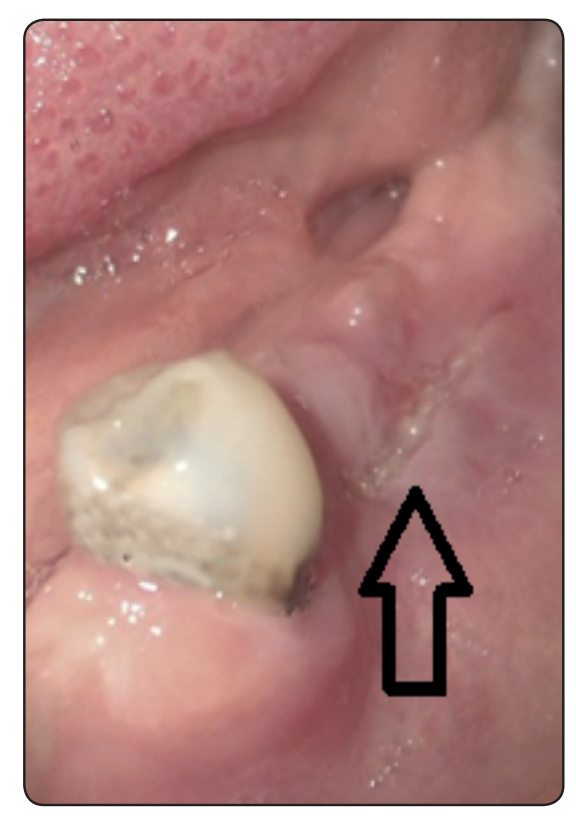

Fig. (4) Showing resolving dehiscence case in group B 3 weeks post-operative

\section{Radiographic results (chart 1)}

Group A showed that the average amount of alveolar bone augmentation gain by fixation technique was $63.4 \% \pm 5.7 \%$ of the pre-operative alveolar bone height (immediately post operative), while the average amount of bone loss (Mean) in the augmented segments after 4 months healing period was $37.893 \% \pm 2.75 \%$ of the amount of augmentation done for the segments of that group. Group B showed an average gain immediately post operative of $74.9 \% \pm 6.4 \%$ of the preoperative ridges vertical heights and an average amount of bone loss in the augmented segments after 4 months

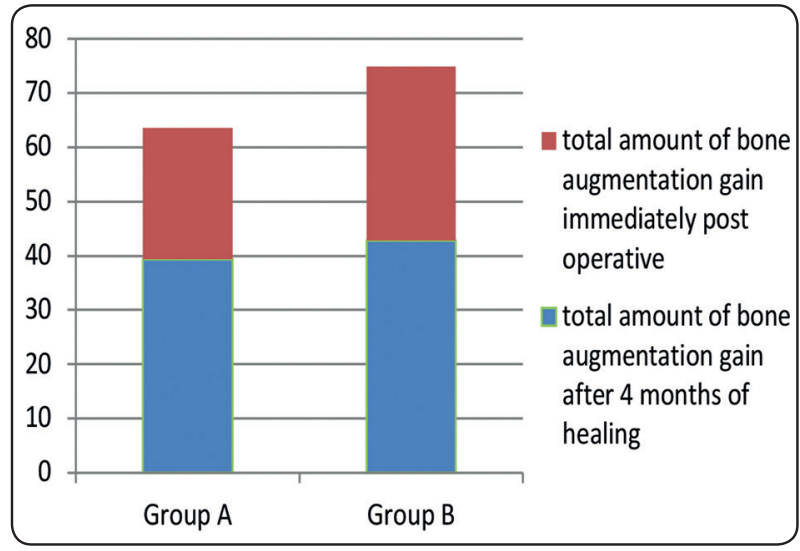

Chart (1) Showing the amount of vertical augmentation achieved in both groups where the $\%$ is relative to that of the actual ridge dimensions (ie. If the pre-operative ridge height was $10 \mathrm{~mm}$ then the augmentation \% was $50 \%$ that means that $5 \mathrm{mms}$ were augmented leaving a total of postoperative $15 \mathrm{mms}$ ridge, while the $\%$ of resorption is to that of the amount of augmentation attained during surgery).

healing period of $42.893 \% \pm 5.2 \%$ of the amount of augmentation achieved. The calculated $\mathrm{P}$ value for the comparison of the amount of graft resorption that occurred in both groups was 0.62 (with statistical significance mark point of $\leq 0.05$ ).

The blue+red columns represent the amount of vertical augmentation achieved immediately post operative, the blue represents the final augmentation $\%$ after 4 months post operative, the red segment means the amount of graft loss.

\section{Histological results}

Histological analysis of the bone specimens taken from the implant osteotomy site of the segments of both groups involving the elevated native alveolar bone and the healed osteotomy area showed:

- The native bone (old bone) was vital, with haversian systems consisting of cylindrical shaped lamellar bone surrounding vascular channels (haversian canals) were evident in specimens of both groups.

- Newly formed bone connected to the old bone by cancellous bone composed of thin bone 
trabeculae and wide marrow spaces. (specimens of group A)

- In group B the newly formed bone (woven bone) appeared more fibrous, stained less intense, the trabeculae appeared less organized and tending to surround large marrow cavities. Some slides contained areas of bone islands surrounded by osteoblasts.

- The presence of osteocytes and osteoblasts was evident, which indicates active bone production in both groups specimens.

- No inflammatory cells were evident in any of the specimens.

Histological examination confirmed the presence of young bone consisting of well observed osteoid, revealing active bone remodeling and new bone apposition to preexisting trabeculae without any evident difference in the specimens of both groups

\section{DISCUSSION}

Block onlay grafts have been used extensively for reconstruction of mandibular alveolar bone defects. Autogenous and non-autogenous bone substitutes are available for vertical and horizontal rehabilitation of deficiencies of the mandible. When a small amount of bone is required, local grafts harvested from the mandibular symphysis or ramus have been extensively used. These sources have the advantage of being convenient because of their proximity to the reconstruction zone and relatively low risk of morbidity. ${ }^{(9,14)}$

In a research evaluating the efficiency of onlay bone grafting techniques in terms of vertical bone formation and dental implant outcomes for correcting atrophied posterior mandibles, it has been stated that a median bone gain of $6.5 \mathrm{mms}$, median bone resorption of $2.75 \mathrm{mms}$ and median finale vertical augmentation of $4 \mathrm{mms}$. So onlay technique resulted in great bone resorption and thus requires the bone block graft to be oversized in height. ${ }^{(14)}$
It has been stated that basic biological principles favor the use of well vascularized flaps to cover the grafted areas. The rationale aims maximize the blood supply to the dependent bone graft and thereby prevent hypoxia with resulting ischemic changes at the distal portions of the flap. ${ }^{(7,8)}$ The most frequently documented complications with bone grafting in literature are surgical wound dehiscence, exposure of the grafts and/or fixation appliances and grafted bone resorption. $(9,10,21)$

In a research evaluating the use of pedicled interpositional grafting technique to for the augmentation of the atrophied posterior mandibles, it has been stated that the overall gain of vertical alveolar height is better achieved with pedicled than with alveolar distraction, that obviously resulted in new bone formation, but could have a large number of drawbacks and an overall complication rate of up to $70 \%$. $^{(15)}$

When inlay grafting is performed height achieved is limited to 6-7 mms. Several explanations for this include that; soft tissue tension has to be kept to minimal in order to allow unobstructed graft healing, so limiting the expansion the vascular blood supply of the raised segments are not put under undesirable strain or stretch which might result in graft mal-perfusion and leads to ischemic resorption of the native mobilized bone graft ,also the bony segment should be stabilized to allow for the incorporation of the graft. Therefore overcorrection with interpositional grafting should be avoided.(20)

As the fixation technique usually requires an additional surgical flap elevation for the removal of the fixation devices, and as that usually will interrupt the subperiosteal plexus of vessels so the blood supply to the crestal bone is there by hindered which could be the explanation for the increased amount of bone resorption compared with the non fixation technique which didn't need additional surgical interventions.

One of the difficulties encountered in non 
fixation technique is the handling of the soft tissues to preserve the blood supply to the mobilized bone segment that should not be put under undue strain or pressure, which in turn limits the amount of vertical and/or horizontal mobilization of the mobilized segment. However it has been stated that as much as $10 \mathrm{mms}$ can be augmented by that technique, in accordance with the results of Marchetti et a1 who showed that 6 to $7 \mathrm{mms}$ of elevation was achievable, Jensen et al who managed 4 to $8 \mathrm{mms}$ and Felice et al stated 4.4 mms. ${ }^{(16-20)}$

Although showing promising results both techniques of interpositional grafting studied in our research showed a relatively noticeable limitation to its clinical application. Where the minimal ridge height that could be operated on was $4 \mathrm{mms}$ that's to avoid any neurosensory affection of the inferior alveolar nerve and to minimize the risk of superior segment fracture. ${ }^{(21)}$ Also the maximum amount of augmentation achievable depends merely on the elasticity of the lingual mucoperiostium on which the alveolar bone segment is pedicled, without guaranteeing the tension and the degree of affection of the lingual pedicle blood supply.

\section{CONCLUSION}

Interpositional grafting technique without the use of a fixation device was a reliable technique for vertical ridge augmentation of the atrophied posterior mandibular ridges with promising rates of success, reduced complications and reduced bone resorption values compared to conventional interpositional grafting with fixation technique.

\section{REFRENCES}

1- Scarano A., Carinci F., Assenza B., Piattelli M., Murmura G. and Piattelli A.: Vertical ridge augmentation of atrophic posterior mandible using an inlay technique with a xenograft without miniscrews and miniplates: case series. Clin. Oral Impl. Res. 22; 1125-1130, 2011.

2- Peter Ehrl, Ulrich Fürst, Arndt Happe, Fouad Khoury, Pavel Kobler, Vitomir Konstantinovic, H.J. Nickenig,
Hakan Özyuvaci, Daniel Rothamel, Witold Tomkiewicz, Jairo Vargas, Andrzej Wojtowicz and Joachim E. Zöller.: Cologne Classification of Alveolar Ridge Defects (CCARD). Consensus paper approved at the 8th European Consensus Conference (EuCC) in Cologne, 9 February 2013.

3- Jose Luis Lopez Cedrtin, MD, DDS, PhD.: Implant Rehabilitation of the Edentulous Posterior Atrophic Mandible: The Sandwich osteotomy Revisited. Int J Oral Maxillofac implants. 26; 195-202, 2011.

4- Truc Thi Hoang Nguyen, Mi Young Eo, Tae Seong Kuk, Hoon Myoung, and Soung Min Kim. Rehabilitation of atrophic jaw using iliac onlay bone graft combined with dental implants. Int J Implant Dent. Dec; 5: 11. 2019.

5- Mohammed S. Abuelnaga, Nader N. Elbokle, Mohammed M. Khashaba. Evaluation of custom made xenogenic bone grafts in mandibular alveolar ridge augmentation versus particulate bone graft with titanium mesh. DOI: 10.21608/ OMX.2018.18827. (2018).

6- Louis P.J., Gutta R. and Said-Al-Naief N.: Reconstruction of the maxilla and mandible with particulate bone graft and titanium mesh for implant placement. J Oral Maxillofac Surg. 66(2); 235-245, 2008.

7- MO Yuce, E Adali, G Turk, G Isik and T Gunbay. Threedimensional bone grafting in dental implantology using autogenous bone ring transplant: Clinical outcomes of a one-stage technique. Nigerian journal of clinical practice. 22(7); 977-981, 2019.

8- Koymen R., Karacayli U., Gocmen-Mas N., ErtugrulKoymen C., Ortakoglu K., Gunaydin Y. and et al. Flap and incision design in implant surgery: clinical and anatomical study. Surg Radiol Anat. 31; 301-306, 2009.

9- Felice P., Pistilli R., Laio G., Pellegrino G., Nisei A. and Marchetti C.: Inlay versus onlay iliac bone grafting in atrophic posterior mandible: a prospective controlled clinical trial for the comparison of two techniques. Implant Dent Relat Res. 11(1); 69-82, 2009.

10- Li J. and Wang H.L.: Common implant-related advanced bone grafting complications: classification, etiology, and management. Implant Dent. 17; 389-401, 2008.

11- Jensen O.T., Kuhlke L., Bedard J.F. and Rosetto D.: Alveolar segmental sandwich osteotomies for vertical anterior maxillary augmentation prior to implant placement. Oral MaxillofacSurg. 64; 290-296, 2006. 
12- Hanle F.: Visor osteotomy to increase the absolute height of the atrophic mandible. A preliminary report. J Oral Maxillo-fac Surg. 3; 257-260, 1975.

13- Schettler D. and Holtermann W.: Clinical and experimental results of a sandwich-technique for mandibular alveolar ridge augmentation. J Maxillofac Surg. 5; 199-202, 1977.

14- A.Sethi, T.Kaus, J.I.Cawood, H.Plaha, M.Boscoe and P.Sochor. Onlay bone grafts from iliac crest: a retrospective analysis. International Journal of Oral and Maxillofacial Surgery. https://doi.org/10.1016/j.ijom.2019.07.001 (2019).

15- Vannucci, Marcello and Doutor, and Em, Especialista and Buco, Cirurgia and Especialista, Maxilofacial and Prótese, Em and Aiquel, Louise and Gaieta, Marcello. Distractions Osteogenesis-An Overview of Challenges and Possibilities. Oral Health and Dentistry Review Article. 2018.

16- Jose Luis Lopez Cedrtin: Implant Rehabilitation of the Edentulous Posterior Atrophic Mandible: The Sandwich osteotomy Revisited. Int J Oral Maxillofac implants. 26; $195-202,2011$.

17- Marchetti C., Trasarti , Corinaldesi G. and Felice P.: Interpositional bone grafts in the posterior mandibular region:
A report on six patients. Int $\mathrm{J}$ Periodontics Restorative Dent. 27; 547-555, 2007.

18- Mario Santagata, Nicola Sgaramella, Ivo Ferrieri, Giovanni Corvo, Gianpaolo Tartaro, and Salvatore D'Amato. Segmental sandwich osteotomy and tunnel technique for three-dimensional reconstruction of the jaw atrophy: a case report. Int J Implant Dent. Dec; 3: 14, 2017.

19- Felice P., lezzi G., Lizio G., Piatelli A. and Marchetti C.: Reconstruction of atrophied posterior mandible with inlay technique and mandibular ramus block graft for implant prosthetic rehabilitation. J Oral Maxillofac Surg. 67; 372380, 2009.

20- S. Titsinides, G. Agrogiannis and T. Karatzas. Bone grafting materials in dentoalveolar reconstruction: A comprehensive review. Jpn Dent Sci Rev. 55(1): 26-32, 2019.

21- Cohen, Adir and Lavi, Dr and Alterman, Michael and Casap, Nardy. Non fixated Inlay Graft For Anterior Localized Mandibular Defect. Oral Surgery, Oral Medicine, Oral Pathology and Oral Radiology. 10.1016/j. oooo.2019.08.010. (2019). 